\title{
The fine-grained metaphysics of artifactual and biological functional kinds
}

\author{
Massimiliano Carrara • Pieter E. Vermaas
}

Received: 7 December 2007 / Accepted: 11 April 2008 / Published online: 21 May 2008

(C) The Author(s) 2008

\begin{abstract}
In this paper we consider the emerging position in metaphysics that artifact functions characterize real kinds of artifacts. We analyze how it can circumvent an objection by David Wiggins (Sameness and substance renewed, 2001, 87) and then argue that this position, in comparison to expert judgments, amounts to an interesting fine-grained metaphysics: taking artifact functions as (part of the) essences of artifacts leads to distinctions between principles of activity of artifacts that experts in technology have not yet made. We show, moreover, that our argument holds not only in the artifactual realm but also in biology: taking biological functions as (part of the) essences of organs leads to distinctions between principles of activity of organs that biological experts have not yet made. We run our argument on the basis of analyses of artifact and biological functions as developed in philosophy of technology and of biology, thus importing results obtained outside of metaphysics into the debate on ontological realism. In return, our argument shows that a position in metaphysics provides experts reason for trying to detect differences between principles of activities of artifacts and organs that have not been detected so far.
\end{abstract}

Keywords Metaphysics - Ontological realism - Artifacts · Artifact functions · Metaphysics of artifacts · Biological organs · Metaphysics of biology · Biological functions · Functional kinds · David Wiggins

\footnotetext{
M. Carrara

Department of Philosophy, University of Padova, P.zza Capitaniato 3, 35139 Padova, Italy e-mail: massimiliano.carrara@unipd.it

P. E. Vermaas $(\varangle)$

Department of Philosophy, Delft University of Technology, Jaffalaan 5, Delft 2628 BX,

The Netherlands

e-mail: p.e.vermaas@tudelft.nl
} 


\section{Introduction}

Artifact functions have recently found their way into the debate on ontological realism. The orthodox position, taken by David Wiggins (1980, 2001, pp. 91-95), for instance, is to reject that artifact functions characterize real kinds of artifacts. This rejection is related to a generally shared Aristotelian anti-realistic conception of artifactsmetaphysically there are not such things as cars and tables because, in an Aristotelian vein, cars and tables do not have their own essences or principles of activity-meaning that artifact functions, or any other feature of artifacts, are not candidates of being essences or principles of activity of artifacts. ${ }^{1}$ Yet, despite this past consensus Lynne Rudder Baker (2004, 2007), Crawford Elder (2004), and Amie Thomasson (2006, 2007a,b) now take artifact kinds as real kinds, where in particular Baker and Elder identify artifact functions as essences of those kinds.

In this paper we analyze this emerging metaphysical role of functions and derive consequences of taking artifact functions and also biological functions as characterizing real kinds. We do so on the basis of current philosophical accounts of artifact functions and biological functions.

Our starting point is Wiggins' original objection to taking artifactual functional kinds as real kinds. The objection is that artifacts for which experts have determined different principles of activity can have the same artifact function, meaning that artifact function is a term that picks out real kinds of artifacts in a too coarse-grained manner. Given their position that artifact functions do characterize real kinds, Baker and Elder have to circumvent this objection. We analyze how this can be done and then show that the resulting metaphysics puts Wiggins' original reason to reject taking artifactual functional kinds as real kinds upside-down: artifacts for which experts have determined the same principle of activity can have different artifact functions, meaning that artifact functions are picking out real artifact kinds in a fine-grained manner.

These consequences generalize to other authors taking this position, and, more surprisingly, generalize also to the biological counterpart of this position, i.e., to the position that biological functions characterize real kinds. In a nutshell the consequences are that taking functions_-artifactual or biological alike-as characterizing real kinds leads to more fined-grained distinctions between real kinds than can be made on the basis of current expert judgments.

The second generalization counters an asymmetry between the metaphysical appreciation of artifact functions and of biological functions. Wiggins again exemplifies this asymmetry since he took biological functions as part of the essences of organ kinds, yet rejected the parallel position for artifact functions (2001, pp. 86-87). The consequences we derive hold, however, for both artifactual and biological functional kinds. Hence, if these consequences are reason to return to the original aversion to artifact functions in metaphysics, they should lead to an equal aversion to biological functions. Or, phrased in positive terms, our argument shows that taking functions as characterizing real kinds will lead in both technology and biology to equally interesting metaphysical results.

\footnotetext{
1 Recent neo-Aristotelian conceptions of artifacts have been formulated by Van Inwagen (1990) and Hoffman and Rosenkrantz (1997).
} 
In Sect. 2 we introduce the relevant key concepts of ontological realism and discuss Wiggins' views on artifact functions and biological functions. At the end of that section, in Sect. 2.4, we give the general structure of our argument that taking functions as characterizing real kinds leads to more fined-grained distinctions between real kinds than can be made on the basis of current expert judgments. In Sect. 3 we run the argument for the artifactual realm for each of the four main accounts of artifact functions. And in Sect. 4 we run it for biology for each of the five main approaches to understanding biological functions. Conclusions follow in Sect. 5.

\section{Ontological realism}

\subsection{Realism about natural kinds}

Ontological realism is - in its minimal characterization - the thesis that something exists in the world that is independent of all our mental states. Usually, this kind of realism (see for example Devitt (1991, p. 17)) is considered of little interest. In fact, it is too weak to support a connected thesis of epistemological realism, i.e., the view that an agent could discover some properties of the world that are independent of the agent's mental states. For if there was no pre-existing structure of the world, all the supposed discoveries of it would be done by mere imposition from an agent. So, a robust ontological realism is thought to require that there exists a world, and such a world possesses a certain structure independent of our beliefs. The world consists of a structured set of entities, called real kinds.

In a robust ontological realism real kinds are ontological "cookie cutters." A real kind collects objects that have a common nature: real kinds partition the world into the discrete particulars that are their instances. For example, real kinds cut the world into human beings, individual dogs, and so on. As a result real kinds $K$ provide us with identity criteria expressing the natural boundaries of those kinds $K$.

Wiggins advances this last position and adds detail to it. For Wiggins the connection between objects of a certain kind and identity criteria is that objects of a certain kind have their identity criteria essentially. Specifically, an identity criterion for a certain kind $K$ of objects gives for things of that kind an answer to the Aristotelian question of "what it is." And because giving a good answer to this Aristotelian question means specifying the essence of a thing, an identity criterion for a certain kind of objects isolates what they are essentially.

Of course, if we accept a robust ontological realism such that a world possesses a certain structure independently of our beliefs, then identity criteria need not be known: the conditions determining whether or not an object belongs to a kind $K$ are independent from the fact that someone accepts those conditions. That means that we have given here an ontological analysis of identity criteria, and not an epistemological one. We are not focusing on the epistemic question of how one can know whether an object $a$ is a $K$, and of how one can know whether $a$ is identical to $b$. Instead we are here interested in providing an ontological analysis that aims at giving necessary and sufficient conditions for $a$ to be a $K$ and for $a$ to be identical to $b$. So, for example, from this point of view it does not suffice to take a man as a human being when s/he has a 
passport, and to take two men as identical if and only if they have the same passport; in Wiggins' terms, these criteria do not give an answer to the Aristotelian question of what a man is.

Secondly, when we speak of identity criteria we have in mind principles such as "material objects are identical if and only if they occupy the same place at all times." That is, we have in mind principles by which we can distinguish a cat from a dog, can identify the cat as the same cat, and can identify the cat as different from another cat. Identity criteria give us a way for distinguishing and counting objects falling under a certain kind $K$.

An identity criterion is thirdly — and sometimes in part- "a vehicle for the articulation of a view concerning what it is for an entity to belong to the kind for which it is given" (Lombard 1986, p. 25). In fact, an identity criterion expresses an essential property of the entities for which the criterion is given. If an identity criterion for a certain kind of objects isolates what essentially they are, we have, for example, an answer to the following question: given that John is a man why could John not have been a cat instead of a man? Following the above sketched strategy an answer to the above question is: Man and cat supply two different identity criteria. John could have been a cat only if he could have had the cat identity criterion in addition to the man identity criterion he has. But identity criteria isolate what essentially he is. Therefore, John could not have that additional 'cat' identity criterion, and thus could not have been a cat.

In ontological realism natural kinds, such as cats and elms, are generally taken as real kinds because criteria exist for such kinds that can be taken as their identity criteria: they give essences of the objects of natural kinds, they allow to distinguish and to count such objects, and they tell what objects such as cats and elms are.

Finally, identity criteria are grounded in what Wiggins calls a "principle of activity". In Wiggins' words, principles of activity are "law-like norms of starting to exist, existing, and ceasing to exist by reference to which questions of identity and persistence can be arbitrated" (Wiggins 2001, p. 83). The determination of natural kinds stands of falls with the existence of those law-like principles, principles that may or may not be known.

In order to specify the nature of the principle of activity Wiggins explicitly refers to some Aristotelian and Leibnizian passages:

Things which exist by nature. . .such as animals and the organs of these or plants and the elementary stuff. . have in them a principle of change or rest (in respect of place or growth and decline or alteration generally)... the nature of a thing being the source or cause of non-accidental change or rest. . (Aristotle Physics Book II, Ch. I)

[D]ivine law once established. . .has truly conferred upon [things] some created impression which endures within them, or... an internal law from which their actions and passions follow. . .if the law of God does in fact leave some vestige of him expressed in things. . .then it must be granted that there is a certain efficacy residing in things, a form of force such as we usually designate by the name of nature, from which the series of phenomena follows (Leibniz 1969, p. $499 \mathrm{ff}$ ). 
According to Wiggins we can think of an activity as a chain of internal and/or external causal interactions, a process determining the persistence of the object. So, for example, life, or perhaps more specifically, the metabolic processes of a human body are processes we can describe using law-like sentences.

Wiggins' proposal resumes Putnam characterization of natural kind terms: $x$ is an $F$ where ' $F$ ' is a natural kind term if and only if, given good exemplars of the kind in question, the best theoretical description that emerged for inquires into kind would group $x$ among the exemplars. Scientist or, more generally, experts make the characterizations of these good exemplars. They give us our best theoretical description. Ordinary speakers defer to experts the application of natural kinds terms because experts known the criteria for the application of such terms. A process of structured cooperation between language-users demarcates the extension of a kind-name in the community language. The idea of semantic deference to experts is practiced-for Putnam - not only with natural kind terms but also with many other sorts of terms, for example artifact terms. ${ }^{2}$

Summarizing, identity criteria for objects of a certain kind $K$ are supposed to do the following four tasks:

1. give a common answer to the question of "what it is" for objects of kind $K$;

2. give principles for distinguishing and counting objects of kind $K$;

3. isolate what objects of kind $K$ are in terms of their essence;

4. express a "principle of activity" of objects of kind $K$, where a principle of activity consists of law-like norms of starting to exist, existing, and ceasing to exist.

Terms that supply in this way identity criteria are called "sortal terms" and examples are natural kinds terms such as "cat" and "elm".

\subsection{Anti-realism about artifactual kinds}

For Wiggins natural kinds are real kinds because natural kinds have identity criteria that isolate the essences of these kinds of objects (i.e., tasks 2 and 3 in the list given at the end of the previous section) via the determination of principles of activity for objects of natural kinds (task 4), thus answering the Aristotelian question of what these objects are (task 1).

Artifactual kinds are for Wiggins, however, not real kinds; the kinds artifacts belong to are for him just conventional ones. Wiggins' reason for this conclusion is that it is not possible to formulate principles of activity for artifactual kinds analogous to those for natural kinds: artifacts can persist through radical mereological changes, interruption of functioning, complete dismantling, and rebuilding. A bicycle, for example, persists even if most of its parts are changed, or if it is during a week spatially scattered and reassembled later on. Wiggins explicitly argues, moreover, that functions of artifacts cannot be considered as providing identity criteria for artifact kinds by denying functions to be the essences of artifacts: "[c]locks, for instance, may be made of a variety of different kinds of material and may function by radically different kinds of

2 On the linguistic labor division see Putnam (1975). 
mechanisms" and "are collected up not by reference to a theoretically hypothesized inner constitution but under functional descriptions that have to be indifferent to specific constitution and particular mode of interaction with environment." (2001, p. 87). According to Wiggins there are thus lots of different devices performing a specific artifact function, and these devices have different internal structures and, according to expert judgment, different principles of activity. Therefore we cannot find regularities in behaviors and form in functionally characterized artifacts, such as clocks, and are they not subject to common laws comparable to the natural kind case. Functional descriptions thus do not refer to an inner constitution of artifacts. Or, phrasing Wiggins' objection differently, functions are too coarse-grained descriptions to perform the four tasks identity criteria are meant to be doing.

The result is twofold. From an epistemological point of view the conclusion is that artifact kinds do not support induction at all. We cannot infer, for example, any truth about chairs from the observation of some instances of chairs. Of course, one could argue that some induction is supported, but that concerns just the nature of the stuff composing the artifacts. From an ontological point of view the result is that artifacts exist only in what Sellars (1963) calls the "manifest image". People project artifact careers, but by a serious ontological inventory of the world artifacts do not exist. In ordinary language people seem to describe a world inhabited by entities of different kinds, and some of them are artificial kinds-tables, computers, clocks, and so on. We utter sentences such as $(i)$ "there is a table in the kitchen," which contain explicit existential idioms and which therefore seem to commit us to the existence of the corresponding entities. Moreover, even without explicit quantification, the very use of a term (singular or general) naturally suggests the existence of a corresponding entity, as in (ii) "the table is heavier than the sofa." Some would say that sentences such as these imply the existence of the entities named. Others would say that they presuppose the existence of those entities. But, in the "manifest image view" ordinary sentences such as ( $i$ ) and (ii) are ontologically opaque; they disguise the true metaphysical make-up of the entities they are about. Consider a philosopher who believes exclusively in the existence of subatomic particles (for example, because this belief complies with a good theory of the physical world, or for some other independent reason). For this philosopher sentences such as (i) and (ii) are, literally taken, not true. If subatomic particles is all there is, then macroscopic objects such as tables and sofas cannot be part of the furniture of the world-hence a fortiori there cannot be tables or sofas.

\subsection{Realism about biological functional kinds}

Wiggins applies the thesis that natural kinds are real kinds also to organ kinds. In fact, the account of natural kinds as real kinds in terms of a principle of activity is according to him extendable to kinds for organs: "the claims so far made", Wiggins argues, referring to natural kinds, "would also apply to words for the natural organs, heart, foot, brain" (2001, p. 86). There is again an Aristotelian debt in the last thesis, as can be noted in the passage Wiggins used: "[A]nimals and the organs of these", Aristotle argues, "have in them a principle of change or rest. . the nature of a thing being the source or cause of non-accidental change or rest..." (Physics Book II, Ch. I). 
More systematically Wiggins distinguishes two types of real kinds: natural kinds, which are determined by a principle of activity, and kinds of biological entities, which are determined by a principle of activity and a mode of functioning. ${ }^{3}$ He argues that $K$ is an organ kind only if $K$ determines a principle of activity and a mode (or principle) of functioning. In analogy to the natural kind case, a principle of activity and of functioning gives for organs an answer to the Aristotelian question of what they are. An identity criterion of an organ kind is isolated via the determination of the principles of activity and functioning of objects of that kind, such that the essence of organs are in part specified by their functions (2001, pp. 86-87). "To be a heart an organ need only be an organ of some definite physiological kind or other playing a particular role in the circulation of the blood" (2001, p. 87 footnote).

So, Wiggins does take biological functions as in part characterizing real kinds in biology and did not raise his 'too coarse-grained' objection to functions within the biological realm. This asymmetry in the metaphysical appreciation of functions in technology and biology, is part of Wiggins' Aristotelian perspective on artifactual and natural kinds. In the same vein, Hoffman and Rosenkrantz in their book on substance (1997) reject the hypothesis that cars and tables exist but, on the contrary, they think that living organisms exist because they have a functional unity. The analysis they offer of organic life involves an evolutionary account of the notion of a natural function.

\subsection{Fine-grained functional and functional ${ }^{+}$kinds}

The noted emergence of artifact functions in ontological realism in the work of Baker (2004, 2007) and Elder (2004) defies the above described asymmetry but also raises the question of how Wiggins' 'too coarse-grained' objection is to be circumvented. An obvious way of doing this is to characterize identity criteria for artifacts that consist of conjunctions of artifact functions and other features of artifacts, such that these criteria single out artifact kinds that do not lump together artifacts with different internal structures and different principles of activity. Following, for example, Wiggins' (2001, p. 100) suggestion one could try to give identity criteria of artifacts in terms of artifacts functions and the notion of 'amount of matter', as formulated by Cartwright (1970). Yet, one can have serious doubts that amount of matter could be regarded as a sortal concept if clock and ship cannot. A better option is to characterize identity criteria for artifacts as a combination of their functions and their 'operational principles' and 'normal configurations'. These latter two notions are defined in engineering-we briefly introduce them in Sect.3 - and with them different artifact internal structures and principles of activity are distinguished. This second option proves that Wiggins' 'too coarse-grained' objection indeed can be circumvented by characterizing identity criteria for artifacts as conjunctions of their functions and other artifact features. But, for keeping our argument as general as possible we do not claim that Baker and Elder have to take this particular solution; other conjunctions may do the trick as well. So, let us refer to this conjunction in general as the "function" ${ }^{+}$of an artifact, where the

\footnotetext{
3 A third kind of entities, expressed by geographical or geological terms, is not considered here.
} 
" + " denotes whatever is added to the function, and let "functional ${ }^{+}$kinds" be the kinds characterized by taking functions ${ }^{+}$as the essences of artifacts.

We argue in the next section that this solution may overshoot its mark in a way that is actually quite interesting: when artifactual functional kinds or functional ${ }^{+}$kinds are taken as real kinds, then artifacts that experts consider as sharing the same principle of activity, say, because they have the same technological structure, may have different artifact functions, thus be of different real kinds, and thus have actually different principles of activity. This discrepancy is not revealing an inconsistency in taking artifactual functional kinds or functional ${ }^{+}$kinds as real kinds, but merely demonstrates the difference between ontology and epistemology: ontological distinctions between real kinds characterize corresponding distinctions between principles of activity, and experts may or may not have knowledge of (all) these distinctions. Yet, and this is the interesting part, ontology may now provide experts reasons as well as indications to start looking for these differences. We run this argument on the basis of current accounts of artifact functions. These accounts are, however, not advancing artifact functions as (partial) essences of artifacts; that assumption is one made by authors in metaphysics such as Baker and Elder.

As said, Wiggins did not raise his objection to taking functional kinds as real kinds in biology. Yet our argument can without problem be run in the domain of biology as well: in Sect. 4 we show that when biological functional kinds or functional ${ }^{+}$kinds are taken as real kinds, then organs that experts may consider as sharing a principle of activity, say, because they have the same morphological structure, may have different biological functions, be of different real kinds, and thus have actually different principles of activity that experts not yet distinguish. We run this argument on the basis of current accounts of biological functions.

Our argument is not primarily meant as a criticism of Wiggins' asymmetric metaphysical treatment of functions in biology and technology. Our argument may be taken as showing that Wiggins could have treated biological and technical functions more symmetrically. But that is not establishing that he should: Wiggins may have other reasons than his 'too coarse-grained' objection for considering organ kinds as real kinds with biological functions being parts of their essences while not considering artifact kinds as real kinds and thus denying artifact functions the metaphysical status of being partial essences. Our points with respect to Wiggins are rather the following. First, his 'too coarse-grained' objection against taking artifact functions as partial essences of artifacts can be circumvented by authors like Baker and Elder, who do take artifact functions as such essences. Second, this circumvention, consisting of taking conjunctive artifact functions ${ }^{+}$as essences of artifacts, leads to an interesting consequence about artifact kinds: artifacts that experts may consider as sharing a principle of activity, can be of different real kinds, and thus have actually different principles of activity that experts not yet distinguish. This consequence holds also for biological organ kinds when biological functions ${ }^{+}$are the essences of organs, as is the case in Wiggins' metaphysics. Hence, also in his metaphysics it is the case that organs that experts may consider as sharing a principle of activity, can be of different real kinds, and thus have actually different principles of activity that experts not yet distinguish.

One may conclude that these points show that Wiggins is right anyway, but should have phrased his original objection against the position of taking artifact functions 
as the essences of artifacts as a " "too fine-grained' objection:" artifacts that have the same principles of activity by expert judgment are on this position divided in different kinds. If this conclusion is to be drawn, then our argument in this paper shows that it is be drawn also for the position of taking biological functions as the essences of organs: this second position, which Wiggins takes, then also leads to a too fine-grained division of organ kinds relative to expert judgment. Yet both conclusions presuppose that expert judgment will not be refined at a latter point in time. This presupposing need not hold, and seems in general also difficult to defend since experts typically can refine their judgments. And that brings us to the interesting part of our argument and the more general point we are making. The metaphysical position of taking functional or functional ${ }^{+}$kinds as real kinds leads to a fine-grained metaphysics and not to a too coarse-grained one, as may be expected from Wiggins' objection: by taking functional or functional ${ }^{+}$kinds as real kinds, distinctions between principles of activity are put forward that are not yet made by experts. Moreover, this point does not hold for the domain of artifacts only but also for the one of biology: the metaphysics of functional and functional ${ }^{+}$kinds is equally interesting in biology and technology.

\section{Artifactual functional and functional ${ }^{+}$kinds}

We first give our argument that taking artifactual functional kinds or functional ${ }^{+} \mathrm{kinds}$ as real kinds may lead to more fine-grained distinctions between principles of activity than experts in technology-that is, engineers - make.

The structure of the argument is as follows. A consequence of the position that artifactual functional or functional ${ }^{+}$kinds are real kinds is that two artifacts that have different functions are not of the same real kind. Which functions artifacts have is determined by the account of artifact functions that holds true. Currently there are by and large four of such accounts available and we consider them all. For each account we choose two artifacts for which it can be made plausible that experts take them as sharing the same law-like norms of starting to exist, existing, and ceasing to exist, and thus as sharing the same principle of activity. Then we show that these two artifacts have different functions, meaning that in the considered position they are of different real kinds and thus have in ontological realism different principles of activity.

The four accounts of artifact function that we consider are listed below. These accounts are the main candidates discussed in philosophy of technology and we therefore limit ourselves to them, conjecturing that our argument holds also with possible other accounts. ${ }^{4}$

4 We, for instance, do not consider the ICE-function theory (Houkes and Vermaas 2004; Vermaas and Houkes 2006). One reason for this is that this theory is explicitly one in which artifact functions are labels that agents ascribe to artifacts; artifact functions are thus not properties that pertain to artifacts and are in this sense less suitable for singling out real artifact kinds. It may, nevertheless, be argued that the ICE-theory can be reformulated as one in which artifacts have their functions as properties (Vermaas 2008). Our argument still holds for such a fifth account of artifact functions: an early-twentieth-century tablet of Aspirin and a current one have different functions in this reformulated ICE-theory, whereas experts plausibly take these tablets as sharing the same law-like norms of starting to exist, existing, and ceasing to exist (the Aspirin example is introduced in Sect.3.3). 
1. The designer intentions account, in which the technical functions of an artifact are the capacities or goals for which agents designed the artifact. $^{5}$

2. The user intentions account, in which the technical functions of an artifact are the capacities or goals for which agents use the artifact. ${ }^{6}$

3. The causal-role account, in which the technical functions of an artifact are the capacities by which it causally contribute to capacities of larger more complex systems. $^{7}$

4. The etiological account in which technical functions of an artifact are the capacities for which the artifact is reproduced in a long-term sense. ${ }^{8}$

For the sake of argument we assume that all these accounts are acceptable, say, by being clear enough and free of contradiction.

The next question to address is the one of when experts judge that two artifacts are sharing the same law-like norms of starting to exist, existing, and ceasing to exist. A strict criterion for this judgment is physical identity: the two artifacts should share an identical physical make-up. A more liberal criterion for experts judging two artifacts as sharing the same law-like norms is technological identity, spelled out as that the two artifacts share the same operational principle and the same normal configuration. The notion of the operational principle of an artifact originates from Michael Polanyi and is described as "how its characteristic parts ... fulfil their special function in combining to an overall operation which achieves the purpose of the" artifact (1958, p. 328). An example is the operational principle of an airfoil of a plane to, "by virtue of its shape, in particular its sharp trailing edge, [generate] lift when inclined at an angle to the airstream" (Vincenti 1990, p. 209). The notion of the normal configuration for an artifact originates from Walter Vincenti-an aeronautic engineer-and is described as "the general shape and arrangement that are commonly agreed to best embody the operational principle" of the artifact. An example is now the normal configuration of automobiles consisting of "four (as against possibly three) wheels and a front-mounted, liquid-cooled engine" (1990, pp. 209-210). Technological identity is supposed to be more liberal since sharing the same operational principle and normal configuration, does not imply sharing the same physical structure: two airplanes that are physically different say by containing a different number of seats may still be sharing their operational principle and normal configuration.

The criterion we favor in this paper is the strict one of physical identity, but at one point in our argument we acknowledge that we may need the more liberal one.

With all these elements in place we now run the argument for each account of artifact functions.

\footnotetext{
5 E.g., Philip Kitcher (1993).

6 E.g., Neander (1991b) and McLaughlin (2001). Note that in these accounts one can take the designer of an artifact as one of its users, making these accounts to subsume the designer intentions account.

7 Cummins (1975).

8 E.g., Millikan $(1984,1993)$ and Preston (1998). Some proponents of the etiological account of functions, specifically Neander (1991b), have proposed that in this account artifact functions are the capacities for which artifacts are reproduced in a short-term sense. This alternative is in our list subsumed under the user intentions and designer intentions accounts.
} 


\subsection{The designer intentions account}

Consider the first phone designed by Alexander Graham Bell, and the later phones that succeeded it. In the designer intentions account of artifact functions the first telephone has the function of aiding the hard-of-hearing, since history has it that Bell designed his original phone for that capacity. Later phones designed by Bell or by others were however designed for long-distance communication and thus have this type of communication as their function in the designer intentions account. The first phone is thus of a different functional or functional ${ }^{+}$real kind than the successor phones. Given the later developments in telephone technology, the consequence that Bell's original phone and a modern twenty-first-century cellular phone are not of the same real kind, may be taken as acceptable: they have different principles of activity, for instance. Yet, on the designer intentions account already the direct successors of Bell's original phone may come out as being of a different kind than the original, and thus as having in ontological realism a different principle of activity than the original.

It is implausible to assume that Bell's first telephone had exactly the same physical structure as the later phones he designed, meaning that we cannot argue on the basis of physical identity that experts judge these phones as sharing the same law-like norms of starting to exist, existing, and ceasing to exist. Yet, some of these early phones may be taken as technologically identical by having the same operational principle and normal configuration. And with this more liberal criterion we can maintain that experts judge that Bell's first phone and (some of) the later phones share the same law-like norms of starting to exist, existing, and ceasing to exist. In a more hypothetical scenario it may be the case that Bell, when designing the long-distance communication system, simply reapplied his original design of the first phone, in which case the original phone and the later ones have different functions in the designer intentions account, are thus of different kinds, but are taken by experts as sharing the same law-like norms on the basis of physical identity.

\subsection{The user intentions account and the causal-role account}

Consider two lengths of metal pipe, one that is included in a chemical installation for transporting a fluid and a second length that is included to give that same installation structural strength. The first length of pipe has the function to carry the fluid in both the user intentions account and the causal-role account, because that is the capacity for which it is used and the capacity that explains the capacity of the whole installation (which may be the production of petrol). The second length of pipe has, however, the capacity to keep parts of the installation together at their proper place in both accounts, because that is the capacity for which the second length is used and the capacity that explains the proper working of the installation. The two lengths of pipe are thus not of the same functional or functional ${ }^{+}$kind, even though experts would judge on the basis of physical identity that they share the same law-like norms of starting to exist, existing, and ceasing to exist. ${ }^{9}$

\footnotetext{
${ }^{9}$ For the user intentions account and the causal-role account one can also show the incoherence between

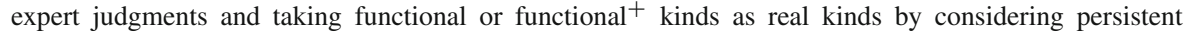




\subsection{The etiological account}

Consider two tables of Aspirin. ${ }^{10}$ The first is produced in the first half of the twentieth century and the second is produced in the twenty-first century. In the etiological account in which artifact functions of artifacts are the capacities for which artifacts are reproduced, the first tablet has pain-killing as its function since at that time this capacity of Aspirin was known and the capacity for which tablets of Aspirin were reproduced. A tablet of Aspirin produced nowadays has the function of pain-killing and blood-clot prevention, since this new capacity has been recently discovered and turned into a second reason for reproducing this drug. Hence, the second tablet has pain-killing and the prevention of blood clots as its function, meaning that it is of a different functional or functional ${ }^{+}$real kind that that the first tablet is. Yet, the tablets are physically the same and experts will take both tablets as sharing the same law-like norms of starting to exist, existing, and ceasing to exist (although those norms may not have been known in the beginning of the twentieth century).

\section{Biological functional and functional ${ }^{+}$kinds}

Our argument on taking functional or functional ${ }^{+}$kinds in the artifactual domain as real kinds can also be run for the biological domain: taking biological functional kinds or functional ${ }^{+}$kinds as real kinds may lead to more fine-grained distinctions between principles of activity than experts in biology — that is, biologists—make.

The structure of the argument is as is sketched in the beginning Sect. 3. A consequence of taking biological functional or functional ${ }^{+}$kinds as real kinds is that two biological items that have different functions are not of the same real kind. Which functions items have depend on the account of biological functions that holds true. There are again a number of candidate accounts available and the ones we consider are adopted from Arno Wouters (2005), who in a recent survey divided the accounts of biological functions currently considered into five approaches. For each approach we choose two biological items, which will be organs, or more specifically muscles of birds, for which it can be made plausible that experts will take them as sharing the same law-like norms of starting to exist, existing, and ceasing to exist, and thus

\footnotetext{
Footnote 9 continued

conditions for artifacts. Take, for instance, an old-fashioned flatiron consisting of a flat wedged-shaped lump of iron onto which a handle is attached. As long as such a flatiron is not physically changed, it remains subjected to the same law-like norms of starting to exist, existing, and ceasing to exist. Originally these artifacts were used solely for ironing and that is then their function in the user intentions account and the causal-role account (and in the other two accounts as well). Many probably have not survived the moment at which they become obsolete due to the advent of more modern flatirons, but some still exist today and are currently used as doorstops (this example is adopted from Preston (1998)). By this new use these latter flatirons have in the user intentions account and in the causal-role account (but not in the other two accounts) picked up a new function and lost their original one. Hence, in these two accounts these flatirons have not persisted as artifacts of the same functional or functional ${ }^{+}$real kind, but stopped at some point of time to persist as artifacts of the ironing or ironing ${ }^{+}$kind to start persisting as artifacts of the door-stopping or door-stopping ${ }^{+}$kind.

10 The example has been taken from Houkes and Vermaas (2004).
} 
as sharing the same principle of activity. Then we show that these two items have different functions, are thus of different real functional or functional ${ }^{+}$kinds, and are thus having in ontological realism different principles of activity.

The five approaches to defining biological functions are listed below. ${ }^{11}$

1. The systemic approach, in which a biological function of an item is "the role of that item in bringing about an activity or capacity of a complex system of which that item is a part."12

2. The goal contribution approach, in which the functions of an item are its causal contributions to the maintenance of a 'goal state' of the organism of which it is a part, where this goal state is a state the organism is disposed to achieve or maintain by varying its behavior accordingly (within a certain range of boundary conditions). ${ }^{13}$

3. The life chances approach, in which "the functions of a trait [are] the effects of that trait that contribute to the life chances of its bearers being higher than the life chances of hypothetical organisms in which that trait is replaced by another one." 14

4. The etiological approach, in which "the functions of a trait are past effects of that trait that causally explain its current presence." 15

5. Two non-historical selection theories by Kitcher (1993), in which "the function of a trait at a certain time is the effect for which that trait is selected for at that time," and by Walsh (1996), in which "the function of a trait in a selection regime is the effect for which that trait is selected for in that selection regime."16

We again assume that all the accounts falling under these approaches are clear enough and free of contradiction.

The next question to address is the one of when expert judge that two organs are sharing the same law-like norms of starting to exist, existing, and ceasing to exist. The strict criterion of physical identity seems not to be reasonably available. When considering artifacts this criterion makes sense since, ignoring wear and tear, two artifacts may indeed have a similar physical structure. Organs are however typically different from a physical point of view due to growth and environmental influences. So we move on to a more liberal criterion and that is that experts judge that two organs are sharing the same law-like norms if the two organs are morphologically similar, which

11 We assume completeness of Wouters' (2005) survey, meaning that, together, these five accounts cover the main accounts of biological functions, conjecturing that our argument holds with possible other accounts as well.

12 Wouters (2005, p. 135). This systemic approach is the biological version of the causal-role account of artifact functions introduced in Sect.3. Wouters associates it with Bock and Von Wahlert (1965) and Cummins (1983, ch. 2).

13 Wouters (2005, p. 138). This approach is connected to Nagel (1961), Boorse (1976) and Adams (1979).

14 Wouters (2005, p. 139). The life chances approach is connected to a number of authors, including Canfield (1964, 1965) and Ruse (1971, 1973).

15 Wouters (2005, p. 141). The etiological account of artifact functions as introduced in Sect. 3 is derived from this etiological approach in biology, which is connected to notably Wright $(1973,1976)$ as well as to Millikan (1984, 1993) and Neander (1991a,b).

16 Wouters (2005, p. 144). 
can be spelled out as that the two organs $(i)$ have the same anatomic position within the organisms concerned and (ii) consist of similar biological tissues structured with a similar internal anatomy. These conditions are only sufficient conditions; two organs may also be taken as sharing the same law-like norms when they have, for instance, a different internal anatomy (think of a healthy heart and a damaged or malformed heart).

We also hold that organs of two organisms of different species, where one species evolved out of the other, may sometimes be taken by experts as sharing the same law-like norms of starting to exist, existing, and ceasing to exist. ${ }^{17}$ This last position amounts to invoking the phenomenon of homology. Homology is, however, not sufficient for organs to be judged to have the same principle of activity; we assume that homologous organs of different species are by expert taken as having the same principle of activity if the organs also meet the second condition (ii) of consisting of tissues with the same biological structure ordered in the same internal anatomy. By insisting on this second condition, we avoid taking disparate but homologous organs such as the forelimbs of a mole and of an eagle as identical organs (see, e.g., Neander 2002). Hence, the criterion we adopt for two organs of organisms of different species to be judged by experts as sharing the same law-like norms of starting to exist, existing, and ceasing to exist, is that these organs are homologous and that condition (ii) holds.

With again all elements in place we can now run the argument for each approach towards biological functions.

\subsection{The systemic approach}

The two organs we consider in our argument are drawn from an example mentioned by Wouters (2005, p. 133) that originates with Hinde (1975). This example consists of the wing muscles of a small bird that lives on a stormy isle and the wing muscles of a similar bird that lives on the main land.

Consider these wing muscles on the systemic approach towards biological functions. Assume that the two birds are of the same species and assume that the bird on the main land flies regularly and that the bird on the isle does not have the disposition to fly. We are agnostic about how this case came about, a possible scenario being that the birds of the species considered initially lived all on the main land, that some birds were blown to the isle and then adapted in a number of generations to their new stormy environment by losing the disposition to fly-birds that kept on flying were blown into sea, and did not reproduce consequently. The wing muscles of the bird on the main land then have a role in bringing about the bird's activity/capacity to fly, and this role is thus a function of those muscles on the systemic approach. The wing muscles of the bird at the isle do not have this role since this second bird does not fly, such that its wing muscles do not have this role as their systemic function. Hence, the wing muscles of the two birds have different functions in the systemic approach and

17 The nature of species is controversial in biology and philosophy, specifically its ontological status is problematic if "species" refers to a real category in nature. Here we do not consider the philosophical problems connected to this notion but use it in a non-problematic case. 
are thus of different real functional or functional ${ }^{+}$kinds, whereas these muscles are still morphologically similar and thus share for experts the same law-like norms of starting to exist, existing, and ceasing to exist.

We acknowledge that this and a few of our other cases may be ones that need not actually have happened. Our point is, however, that they are biologically possible and that it is therefore possible that, if one takes biological functional or functional ${ }^{+}$kinds as real kinds, organs that experts consider as sharing a principle of activity, may have in ontological realism different principles of activity.

\subsection{The goal contribution approach}

Both the bird on the main land and the one on the isle have survival and reproduction as their goal states. If we assume that there are predators walking at the ground on the main land but not at the isle, then for the first bird the wing muscles do causally contribute to the maintenance of this goal whereas the wing muscles of the second bird do not. Hence, on the goal contribution approach this contribution is corresponding to a function of the wing muscles of the bird living on the main land, and is not corresponding to a function of the wing muscles of the bird on the isle. The wing muscles of the two birds thus have also different functions in the goal contribution approach. ${ }^{18}$

\subsection{The life chances approach}

To argue for this conclusion in the case of the life changes approach and the etiological approach, we need to make some additional assumptions about our case. We need to assume that the bird on the isle is part of a colony of birds. This colony has been relatively isolated for $n$ generations on the isle, and birds of this colony have during these $n$ generations started to use their wings for new behavior that is different to flying. Let this new behavior be deterrence: both male and female birds flap their wings to scare away other birds when they collect food on the ground. And assume finally that the mainland birds did not pick up this particular behavior, say, because they hardly forage on the ground due to those predators.

The hypothetical organism to which the bird on the main land has to be compared for determining the functions of its wing muscles in the life chances approach, can be a flightless bird. Then it can be argued that the effects of driving flying of the wing muscles are for the bird on the main land typically contributing more to the life chances of the bird—due to those predators- than the effects of the idle wing muscles of the

\footnotetext{
18 The incoherence between expert judgments and taking functional or functional ${ }^{+}$kinds as real kinds can sometimes also be demonstrated by considering persistent conditions for organs, similar to the equivalent phenomenon for artifacts (see the footnote in Sect. 3.2). Consider a bird that is blown at some time $t$ from the main land to the stormy isle. Before $t$ flying did contribute to the maintenance of the goal state of survival and reproduction, whereas after $t$ flying does not. Hence, before $t$ the causal contribution of the bird's wing muscles does correspond to a function of the muscles, whereas it does not after $t$. This means that in the goal contribution approach the muscles before $t$ are of a functional or functional ${ }^{+}$real kind different to the functional or functional ${ }^{+}$kinds it is of after $t$. Yet, from a biological point of view, the law-like norms of starting to exist, existing, and ceasing to exist, of these muscles do not change around $t$. For the life chances approach and the non-historical selection theories a similar argument can be given.
} 
hypothetical bird. Hence, for the bird on the main land driving flying is a function of its wing muscles on the life chances approach. The hypothetical organism to which the bird at the isle has to be compared can in turn be a bird that does not flap its wings when competing with other birds over available food. The argument can now be that the effects of driving flapping of the wing muscles are for the bird on the isle typically contributing more to the life chances of the bird-the flapping secures better access to food - than the effects of the idle wing muscles of the hypothetical bird. Hence, for the bird at the isle driving 'deterrence flapping' may be taken as a function of its wing muscles on the life chances approach, leading again to the conclusion that the wing muscles of the bird living on the main land have different functions than those of the bird living on the isle.

\subsection{The etiological approach}

If the number $n$ of generations at which this colony has been relatively isolated on the isle, increases, then at some point flapping of the wings for deterrence during foraging becomes part of the causal explanation of why the isle birds have their wing muscles. Flying may also be mentioned as an important element in that causal explanation, but it is complemented with 'deterrence flapping.' This means on the etiological approach that the wing muscles of a recent bird on the isle has contributing to deterrence flapping as their functions, whereas on the same approach the wing muscles of a recent bird on the main land do not have this function. The number $n$ being large may mean that from a biological point of view the birds on the main land and on the isle cannot be counted as belong to the same species anymore. The wing muscles of the two birds become then homologues, but can still have the same morphology and in this sense be taken as having the same law-like norms of starting to exist, existing, and ceasing to exist. Yet, the muscles are of different functional or functional ${ }^{+}$real kinds in the etiological approach.

\subsection{The two non-historical selection theories}

Finally, in the case of the two non-historical selection theories, we need not make additional assumptions. The selection regimes that are in place for the wing muscles of the two birds are different since they favor flying on the mainland and not-flying at the isle. This holds at all times, hence on both Kitcher's (1993) theory and Walsh (1996) theory, the functions of the wing muscles of the two birds are different.

\section{Conclusions}

In this paper we have considered the emergence of artifact functions in the debate on ontological realism. The position that artifact functions characterize real kinds of artifacts was rejected by, for instance, David Wiggins (1980, 2001, pp. 91-95) but is now advanced by Lynne Rudder Baker (2004, 2007) and Crawford Elder (2004). We have considered Wiggins' objection to this position consisting of the claim that 
artifact functions leads to a too coarse-grained metaphysics: artifacts about which experts judge that they have different principles of activity, are lumped together in single functional kinds. This objection can be circumvented by characterizing real artifact kinds by means of conjunctions of the artifacts' functions and some other of their features. We denoted those other features with a " + ".

The main argument in the paper is that taking functional or functional ${ }^{+} \mathrm{kinds}$ as real kinds leads actually to a fine-grained metaphysics: by taking functional or functional ${ }^{+}$ kinds as real kinds, distinctions between principles of activity are posited that are not yet made by experts.

We have, moreover, argued that this argument holds for both the domain of artifacts and the domain of biology. Wiggins launched his 'too coarse-grained' objection against taking artifact functions as characterizing real artifact kinds, and not against taking biological functions as characterizing real organ kinds, thus establishing an asymmetry between the metaphysical status of artifact functions within technology and of biological functions within biology. Our argument established that both types of functions are equally interesting from a metaphysical point of view.

We have run our argument on the basis of analyses of artifact functions and biological functions as they have been developed in philosophy of technology and philosophy of biology, thus importing results obtained outside of metaphysics into the debate on ontological realism. More generally, one can interpret the emergence of artifact functions in this debate as an attempt to let the notion of real kinds cohere better with outside experts' points of view. Identity criteria should be compatible with the results of science (see on this point (Savellos 1982, p. 830)) but also with (every-day) technology. Baker (2004, pp. 99-112) defends the thesis that artifacts really exist, against philosophers - from Aristotle, to Leibniz, to Van Inwagen - who find artifacts ontologically deficient. Elder (2004) introduces a naturalistically inclined ontology that includes many common sense objects, such as persons, organisms, and some (kinds of) artifacts. Finally Thomasson (2006) analyses most of the prominent arguments against accepting commonsense objects as tables and chairs, and provides a unified diagnosis of where these arguments go wrong.

Whether this effort to make metaphysics more coherent to science and technology will be successful is to be seen. Wiggins' original argument does not rule out taking functional or functional ${ }^{+}$kinds are real kinds, but other problems may emerge (see, for instance, the analysis by Houkes and Meijers (2006) of Baker's (2004) position). Notably it seems that a tension with the minimal characterization of ontological realism has to be resolved, since in all the accounts considered in Sect. 3, artifact functions depend in some way on mental states of agents, meaning that the existence of functional and functional $^{+}$kinds cannot be in all possible senses independent of our mental states. But even if problems do emerge, the question remains whether they will be reason to stop the mentioned effort. Thomasson (2007a), for instance, has already argued that if artifact kinds do not fit the standard schemes of metaphysics, it may be these schemes that have to give in rather than artifact kinds.

Our argument is in our view not one against taking functional or functional ${ }^{+}$kinds as real kinds. It rather seems to establish a reverse effort from metaphysics towards science and technology. The argument provides experts reasons as well as indications to start looking for differences between principles of activities of artifacts and of organs 
that so far have not been detected. That is, if functional or functional ${ }^{+}$kinds are real kinds, then it may be expected that all kinds of fine-grained distinctions between lawlike norms are to be discovered in technology and biology: artifacts and biological organs that are now typically taken as having the same technological or biological structure, respectively, will have different principles of activity once they have different functions. Also about this reverse effort it does not make sense to predict whether it will be successful. Yet, the mere conceptual possibility that it is successful makes the metaphysics of functions already rather interesting.

Acknowledgements We like to gratefully thank Wybo Houkes, Peter Kroes, Marzia Soavi, Achille Varzi, and two anonymous reviewers for helpful discussions and comments. Massimiliano Carrara is indebted to the Philosophy Department of Delft University of Technology for financial support to realize a preliminary version of this paper. Research by Pieter Vermaas was supported by the Netherlands Organization for Scientific Research (NWO).

Open Access This article is distributed under the terms of the Creative Commons Attribution Noncommercial License which permits any noncommercial use, distribution, and reproduction in any medium, provided the original author(s) and source are credited.

\section{References}

Adams, F. R. (1979). A goal-state theory of function attributions. Canadian Journal of Philosophy, 9, 493-518.

Baker, L. R. (2004). The ontology of artifacts. Philosophical Explorations, 7, 99-111.

Baker, L. R. (2007). The metaphysics of everyday life: An essay in practical realism. Cambridge: Cambridge University Press.

Bock, W. J., \& Von Wahlert, G. (1965). Adaptation and the form-function complex. Evolution, 19, 269-299.

Boorse, C. (1976). Wright on functions. Philosophical Review, 85, 70-86.

Canfield, J. (1964). Teleological explanations in biology. British Journal for the Philosophy of Science, 14, 285-295.

Canfield, J. (1965). Teleological explanations in biology: A reply. British Journal for the Philosophy of Science, 15, 327-331.

Cartwright, H. M. (1970). Quantities. Philosophical Review, 79, 25-42.

Cummins, R. (1975). Functional analysis. Journal of Philosophy, 72, 741-765.

Cummins, R. (1983). The nature of psychological explanation. Cambridge, MA: MIT Press.

Devitt, M. (1991). Realism and truth. Oxford: Basil Blackwell.

Elder, C. (2004). Real natures and familiar objects. Cambridge, MA: MIT Press.

Hinde, R. A. (1975). The concept of function. In G. R. Baerends, C. Beer, \& A. Manning (Eds.), Function and evolution in behaviour (pp. 3-15). Oxford: Clarendon.

Hoffman, J., \& Rosenkrantz, G. S. (1997). Substance: Its nature and existence. London: Routledge.

Houkes, W., \& Meijers, A. W. M. (2006). The ontology of artefacts: The hard problem. Studies in History and Philosophy of Science, 37, 118-131.

Houkes, W., \& Vermaas, P. E. (2004). Actions versus functions: A plea for an alternative metaphysics of artifacts. Monist, 87, 52-71.

Kitcher, P. S. (1993). Function and design. In P. A. French, T. E. Uehling, \& H. K. Wettstein (Eds.), Midwest studies in philosophy (Vol. XVIII, pp. 379-397). Minneapolis: University of Minnesota Press.

Leibniz, W. (1969). On nature itself, or on the inherent force and actions of created things. In Id. Philosophical papers and letters (trans: Loemker, L. E.). Dordrecht: Reidel.

Lombard, L. B. (1986). Events: A metaphysical study. London: Routledge \& Kegan Paul.

McLaughlin, P. (2001). What functions explain. Cambridge: Cambridge University Press.

Millikan, R. G. (1984). Language, thought, and other biological categories: New foundations for realism. Cambridge, MA: MIT Press.

Millikan, R. G. (1993). White Queen psychology and other essays for Alice. Cambridge, MA: MIT Press.

Nagel, E. (1961). The structure of science. London: Routledge \& Kegan Paul. 
Neander, K. (1991a). Functions as selected effects: The conceptual analyst's defense. Philosophy of Science, $58,168-184$.

Neander, K. (1991b). The teleological notion of "function". Australasian Journal of Philosophy, 69, 454-468.

Neander, K. (2002). Types of traits: The importance of functional homologues. In A. Ariew, R. Cummins, \& M. Perlman (Eds.), Functions: New essays in the philosophy of psychology and biology (pp. 390-415). Oxford: Oxford University Press.

Polanyi, M. (1958). Personal knowledge: Towards a post-critical philosophy. London: Routledge \& Kegan Paul.

Preston, B. (1998). Why is a wing like a spoon? A pluralist theory of functions. Journal of Philosophy, 95, 215-254.

Putnam, H. (1975). The meaning of "meaning". In Id. Mind, language and reality: Philosophical papers (pp. 215-271). Cambridge: Cambridge University Press.

Ruse, M. (1971). Functional statements in biology. Philosophy of Science, 38, 87-95.

Ruse, M. (1973). The philosophy of biology. London: Hutchinson.

Savellos, E. (1982). Criteria of identity and the individuation of natural-kinds events. Philosophy and Phenomenological Research, 52, 807-831.

Sellars, W. (1963). Science, perception and reality. London: Routledge \& Kegan Paul.

Thomasson, A. (2006). Metaphysical arguments against ordinary objects. Philosophical Quarterly, 56, 340-359.

Thomasson, A. (2007a). Artifacts and human concepts. In E. Margolis \& S. Laurence (Eds.), Creations of the mind: Theories of artifacts and their representation (pp. 52-71). Oxford: Oxford University Press.

Thomasson, A. (2007b). Ordinary objects. New York: Oxford University Press.

Van Inwagen, P. (1990). Material beings. Ithaca: Cornell University Press.

Vermaas, P. E. (2008). On unification: Taking technical functions as objective (and biological functions as subjective). In U. Krohs \& P. Kroes (Eds.), Functions in biological and artificial worlds: Comparative philosophical perspectives, Vienna series in theoretical biology (in press). Cambridge, MA: MIT Press.

Vermaas, P. E., \& Houkes, W. (2006). Technical functions: A drawbridge between the intentional and structural natures of technical artefacts. Studies in History and Philosophy of Science, 37, 5-18.

Vincenti, W. G. (1990). What engineers know and how they know it: Analytic studies from aeronautical history. Baltimore: Johns Hopkins University Press.

Walsh, D. M. (1996). Fitness and function. British Journal for the Philosophy of Science, 47, 553-574.

Wiggins, D. (1980). Sameness and substance. Oxford: Blackwell.

Wiggins, D. (2001). Sameness and substance renewed. Cambridge: Cambridge University Press.

Wouters, A. (2005). The function debate in philosophy. Acta Biotheoretica, 53, 123-151.

Wright, L. (1973). Functions. Philosophical Review, 82, 139-168.

Wright, L. (1976). Teleological explanations: An etiological analysis of goals and functions. Berkeley: University of California Press. 